\title{
Influence of Martensite Volume Fraction on Mechanical Properties of High-Mn Steel
}

\author{
Rashid Khan, Tasneem Pervez, Sayyad Zahid Qamar \\ Mechanical \& Industrial Engineering Department, College of Engineering Box 33, Sultan Qaboos University, Muscat, Oman \\ Email: rashidkhan.ned@gmail.com
}

Received September 10, 2013; revised October 12, 2013; accepted October 21, 2013

Copyright (C) 2013 Rashid Khan et al. This is an open access article distributed under the Creative Commons Attribution License, which permits unrestricted use, distribution, and reproduction in any medium, provided the original work is properly cited.

\begin{abstract}
Elastic-plastic deformation behavior of austenitic, martensitic, and austenitic-martensitic high-Mn steels is investigated by using crystal plasticity theory. The development of expandable pipes made of two-phase steel for oil and gas well applications is needed for improved and efficient recovery of hydrocarbons from difficult reservoirs. The current research is aimed at improving the down-hole post-expansion material properties of expandable pipes. A mathematical model is first developed based on finite-deformation crystal plasticity theory assuming that slip is the prime mode of plastic deformation. The developed model is then numerically implemented by using the finite element software ABAQUS, through a user defined subroutine. Finite element simulations are performed for austenitic, martensitic, and austenitic-martensitic steels having different proportions of martensite in an austenite matrix. Three primary modes of loading are considered: uniaxial tension, compression and simple shear. The variation in yield strength, hardening pattern and dissipated energy is observed and analyzed.
\end{abstract}

Keywords: Dual-Phase Steel; Martensite; Mechanical Properties; Micromechanical Modeling; Finite Element Method

\section{Introduction}

As easily recoverable hydrocarbon resources are depleting, the oil and gas industry focuses more on exploring oil and gas from ultra-deep, tight and pocketed reservoirs. Not only are these recoveries difficult and costly, but they require the development of new technologies and materials. There is a significant demand for new metallic materials for well drilling and construction that can meet stringent requirements regarding operation in sub-surface environment, elevated strength, immunity to sulphur cracking, and light in weight. This poses new challenges for materials scientist and engineers. This need is a driving force for the development of modern innovative steel grades for oil and gas well applications. One of the new applications in well drilling and remediation is expandable pipes, requiring the development of modern steels. These expandable pipes go through large diametral expansion at depths of several kilometers in onshore or offshore wells. The resulting large permanent deformation alters the post-expansion mechanical properties of the pipes and can lead to premature failure during operations. Since the first innovative development of expandable pipe [1] to the recent developments [2], the challenge of getting all desired material properties is still a distant reality. Although a series of innovative steels (TRIP, TWIP, Dual-phase, Martensitic steel, etc.) are being developed in various research laboratories around the world, the applications are focused more on transportation industry [3]. The idea revolves around complex thermooperations to obtain sophisticated microstructures with combinations of different size grains, multiphase microstructure, and preferred crystallographic orientation. The changes in grain morphology and the distribution of phases at the micro level during manufacturing lead to significant improvement in material properties at macro level. Dual-phase steel is categorized as advanced highstrength steel (AHSS), and has two phases; either austenite and ferrite, or austenite and martensite. Austenite is a high temperature phase but in case of dual-phase steels, metastable austenite exists at room temperature which may transform into martensite upon application of thermo-mechanical loads [4]. The existence of retained austenite plus the transformation mechanism may give rise to the phenomenon of transformation induced plasticity (TRIP), which ultimately enhances the strength and formability of an alloy [5]. It is envisaged that the down- 
hole expansion of pipes in a well can be done in a way to exploit the interaction between micro-phases to elevate its properties after expansion [6]. The elevated properties will enhance structural integrity of the well, collapse and burst strength of the pipe, as well as providing a safeguard against possible mechanical failures such as buckling etc.

Currently, high-Mn steel is used to manufacture expandable pipes consisting mainly of two phases, austenite and ferrite with some traces of martensite. Presence of a reasonable amount of martensite phase will greatly enhance the mechanical properties of the pipe. In other words, if during permanent deformation process of pipes, the cubic austenite phase transforms to tetragonal martensite phase due to the spontaneous shear deformation of crystal lattice by mechanical stressing, then the resulting property of the expanded pipe will be better than preexpanded pipe. Extensive research has been done on martensitic transformation (MT) of steel, both theoreticcally and computationally, but no work has been done with oil well applications in mind. Different types of MT models are developed which span from single to polycrystalline, atomic to continuum, and microscopic to macroscopic [7]. Whether these models are simple or complex, one solution approach is based on the finite element method (FEM). Crystal plasticity FEM is one such technique which is developed to define the complete transformation and deformation characteristics of single or poly-crystal to deform and/or transform from one type of crystal lattice to other. In this work, crystal plasticity theory based on finite element formulation is used to predict the deformation behavior of dual-phase high-Mn steel.

An elastic-plastic deformation behavior of high-Mn steel is analyzed when subjected to external mechanical load. Different proportions of martensite in this steel are considered. The main objective is to estimate the deformation pattern of high-Mn alloy steel having different volume fractions of martensite phase in an austenite matrix. Initially, a mathematical model based on the crystal plasticity theory is presented in order to estimate the elastic-plastic deformation. Then, crystal plasticity model using the time integration procedure is implemented as a user defined material subroutine in the commercial finite element software ABAQUS [8]. Finally, finite element simulations are performed to analyze the stress-strain behavior of high-Mn steel having different percentage of austenite and martensite.

\section{Problem Formulation}

The elastic-plastic deformation behavior of single crystal is modeled using crystal plasticity theory, assuming that slip constitutes the dominant part of the plastic deformation. The kinematics of a single crystal based on the finite deformation theory can be expressed using multiplicative decomposition of total deformation gradient [9] as given by

$$
F=F^{e} \cdot F^{p},
$$

where $F$ represents the total deformation gradient, while $F^{e}$ and $F^{p}$ are the elastic and plastic deformation gradients, respectively. The elastic deformation gradient can be further decomposed into symmetric left stretch tensor and orthogonal rotation tensor:

$$
F=U^{e} R^{e} \cdot F^{p},
$$

where $U^{e}$ is the left stretch tensor while $R^{e}$ is the orthogonal rotation tensor. The elastic deformation gradient $F^{e}$ describes the deformation of a crystal due to elastic stretch and rigid body rotation, while the plastic deformation gradient $F^{p}$ represents only the deformation due to crystallographic slip, which results due to dislocation movement on preferred crystallographic planes termed as slip planes. Crystallographic slip deformation does not alter the lattice structure and thus the elastic properties remain unchanged during the course of deformation. The plastic deformation gradient must satisfy plastic incompressibility condition $\operatorname{det} F^{p}=1$. Therefore the determinant of total deformation gradient will be greater than zero, $\operatorname{det} F>0$. The rotation and plastic deformation gradient tensors can be combined into a plastic rigid rotation tensor $F^{*}$ as

$$
F=U^{e} \cdot F^{*}, F^{*}=R^{e} \cdot F^{p}
$$

Let $\mathrm{B}_{0}$ and $\mathrm{B}_{f}$ represent the reference (undeformed) and current (deformed) configurations of a material point. The decomposition of total deformation gradient can be represented by two intermediate states between these two end configurations, as shown in Figure 1. The two intermediate configurations $\mathrm{B}_{1}$ and $\mathrm{B}_{2}$ represent the states which the crystal experiences to attain the total deformation. The first intermediate state $B_{1}$ represents the con-

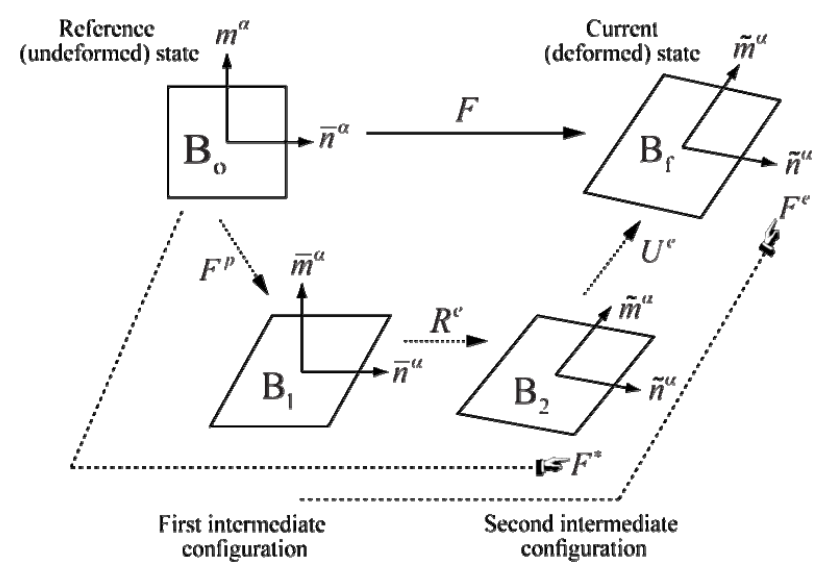

Figure 1. Kinematic decomposition of elastic-plastic deformation of single crystal. 
figuration with full plastic strain, which when subjected to rigid rotation leads to the second intermediate state $B_{2}$. The final deformed state of a material in current configuration $\left(\mathrm{B}_{f}\right)$ is projected from second intermediate state $\mathrm{B}_{2}$ through stretch tensor $U^{e}$.

During elastic-plastic deformation of a crystal in slip mode, the major deformation mode is shear, resulting in plastic flow. This rate of shear strain is termed as plastic velocity gradient $L^{p}$. The plastic deformation gradient can be represented in terms of plastic velocity gradient as

$$
\dot{F}^{p}=L^{p} F^{p},
$$

In a crystal where slip activity happens on a number of crystallographic planes (slip planes), the plastic velocity gradient can be expressed as

$$
L^{p}=\dot{F}^{p}\left(F^{p}\right)^{-1}=\sum_{\alpha=1}^{N_{S L}} \dot{\gamma}^{\alpha} \bar{S}^{\alpha}=\sum_{\alpha=1}^{N_{S L}} \dot{\gamma}^{\alpha}\left(\bar{m}^{\alpha} \otimes \bar{n}^{\alpha}\right),
$$

where $\alpha$ represents a slip system $\left(\alpha=1, \cdots, N_{S L}\right), N_{S L}$ is the total number of slip systems in a crystal, $\dot{\gamma}^{\alpha}$ is the plastic shear strain rate on $\alpha$-slip system, and $\bar{S}^{\alpha}$ is the Schmid orientation tensor in first intermediate configuration. The Schmid tensor is defined by the dyadic product of slip direction vector $\bar{m}^{\alpha}$ and area normal vector of slip plane $\bar{n}^{\alpha}$ as $\bar{S}^{\alpha}=\bar{m}^{\alpha} \otimes \bar{n}^{\alpha}$. The Schmid tensor must be updated in the second intermediate configuration to account for the lattice rotation effects on the deformation behavior of the crystal. A forward mapping function is used to estimate the Schmid tensor in the second intermediate configuration as

$$
\tilde{S}^{\alpha}=R^{e} \bar{S}^{\alpha}\left(R^{e}\right)^{\mathrm{T}}=\tilde{m}^{\alpha} \otimes \tilde{n}^{\alpha},
$$

where $\tilde{S}^{\alpha}$ represents the Schmid tensor in second intermediate configuration $\mathrm{B}_{2}$. The slip direction vector $\tilde{m}^{\alpha}$ and area normal vector of slip plane $\tilde{n}^{\alpha}$ can be estimated as

$$
\tilde{m}^{\alpha}=F^{e} \bar{m}^{\alpha}=U^{e} R^{e} \bar{m}^{\alpha}=R^{e} \bar{m}^{\alpha}, \tilde{n}^{\alpha}=R^{e} \bar{n}^{\alpha} .
$$

A list of slip directions and area normal vectors for face centered cubic (FCC) and body centered cubic (BCC) crystals can be found in [10]. The shear strain rate on $\alpha$-slip system in Equation (4) can be estimated using the specific constitutive power law function [11], as given by

$$
\dot{\gamma}^{\alpha}=\dot{\gamma}_{0}\left|\tau^{\alpha} / s^{\alpha}\right|^{1 / m} \sin \left(\tau^{\alpha}\right),
$$

where $\dot{\gamma}_{0}$ is the initial shear strain rate (constant for all slip systems), $\tau^{\alpha}$ is the resolved shear stress (RSS), $s^{\alpha}$ is the slip resistance for $\alpha$-slip system, and $\mathrm{m}$ is the rate sensitivity parameter for the shear strain. The ratio of RSS and slip resistance in the constitutive formulation of Equation (7) describes the slip activity, which is termed as slip activity ratio, while the sensitivity parameter indicates the response of a slip system subjected to the specific RSS value. Two similar slip systems may not activate at the same magnitude of RSS. The activity of slip system also depends on the slip resistance, which is mainly governed by the orientation of slip plane with respect to the loading axis. Any slip system cannot be activated unless this ratio becomes greater than unity.

The slip resistance $s^{\alpha}$ is responsible for the hardening or softening of a slip system in a crystal at micro level and affects material deformation at macro level. In addition, the slip resistance of an individual slip system mainly depends on the slip activity of other slip systems. For example, the slip resistance of $\alpha$-slip system $(\alpha=i)$ may increase or decrease if it interacts with another slip system $\beta\left(\beta=k, k=1, \cdots, i-1, i+1, \cdots, N_{S L}\right) \quad$ which is inactive or more/less active at the same stress level. This mechanism causes hardening or softening of $\alpha$-slip system. The interaction phenomenon becomes more complex when $\alpha$-slip system may interact with more than one $\beta$-slip systems. Furthermore, the variation in shearing rate of $\beta$-slip systems may cause further complexity in hardening mechanism, which may result in problems of numerical solution convergence. These complexities can be lessened by assuming self-hardening i.e. no interaction among slip systems, and identical slip resistance for all $\alpha$-slip systems. In this work, only identical slip resistance is considered for all slip systems. The slip resistance for $\alpha$-slip system can be expressed as

$$
\dot{s}^{\alpha}=\sum_{\beta=1}^{N_{S L}} h^{\alpha \beta}\left|\dot{\gamma}^{\beta}\right|,
$$

where $h^{\alpha \beta}$ is the strain hardening parameter of $\alpha$-slip system due to the interaction with $\beta$-slip system, and $\dot{\gamma}^{\beta}$ is the shear strain rate of $\beta$-slip system. The strain hardening parameter $h^{\alpha \beta}$ is estimated as $h^{\alpha \beta}=q^{\alpha \beta} h^{\beta}$, where $q^{\alpha \beta}$ represents the hardening coefficient of $\alpha$ slip system. The value of $q^{\alpha \beta}=1.0$ represents selfhardening $(\alpha=\beta)$, while for hardening due to slip systems interaction $(\alpha=\beta), q^{\alpha \beta}>1$. The single slip hardening parameter $h^{\beta}$ can be estimated as

$$
h^{\beta}=h_{0}\left(1-s^{\beta} / s_{\infty}\right)^{b},
$$

where $h_{0}$ is the initial value of hardening parameter, $s^{\beta}$ is the slip resistance of $\beta$-slip system, $s_{\infty}$ is the saturation value of slip resistance, and $b$ is the hardening sensitivity parameter which depends on slip resistance. The hardening parameters $h_{0}, s_{\infty}$ and $b$ are assumed to be constant for all slip systems. Considering the reference configuration, the Green finite strain tensor $E^{*}$ can be calculated from the elastic deformation gradient as

$$
E^{*}=\frac{1}{2}\left[\left(F^{e}\right)^{\mathrm{T}} F^{e}-I\right] .
$$


The elastic deformation gradient can be calculated using Equation (1)

$$
F^{e}=F\left(F^{p}\right)^{-1}
$$

Once Green strain tensor is calculated, the second PiolaKirchhoff stress $T^{*}$ can be obtained using crystal plasticity constitutive equation as given below

$$
T^{*}=\tilde{\mathbb{C}}: E^{*},
$$

where $\tilde{\mathbb{C}}$ is the elasticity tensor. The effective elasticity tensor, $\tilde{\mathbb{C}}^{e}$ for current two-phase high-Mn steel is determined using the elasticity tensors of austenite $\left(\tilde{\mathbb{C}}^{a}\right)$ and martensite $\left(\tilde{\mathbb{C}}^{m}\right)$ based on the rule of mixtures, the most common homogenization technique used in computational mechanics:

$$
\tilde{\mathbb{C}}^{e}=\left[\left(1-\xi^{m}\right) \tilde{\mathbb{C}}^{a}+\xi^{m} \tilde{\mathbb{C}}^{m}\right],
$$

where $\xi^{m}$ represents the volume fraction of martensite. The resolved shear stress on $\alpha$-slip system of Equation (7) can be estimated using Schmid law

$$
\tau^{\alpha}=F^{e} T^{*} \tilde{S}^{\alpha},
$$

where $T^{*}$ is obtained using Equation (12) and other two parameters are already known from kinematic and hardening descriptions. Finally, the true stress $T$ (Cauchy stress) can be calculated as

$$
T=\left(F^{e}\right)\left\{\left(\operatorname{det} F^{e}\right) T^{*}\right\}\left(F^{e}\right)^{\mathrm{T}} .
$$

It is interesting to observe the changes in amount of energy dissipated with increasing volume fraction of martensite during the elastic-plastic deformation of crystal. The extended form of this dissipated energy is given in detail in [12] and is summarized as

$$
D=\sum_{\alpha=1}^{N_{S L}}\left[\left(\tau^{\alpha} \dot{\gamma}^{\alpha}\right)+\left(\Omega^{\alpha} \dot{\gamma}^{\alpha}\right)-\left\{\left(w_{a} \mu_{a} \beta_{a}\right) \omega^{\alpha} \dot{\gamma}^{\alpha}\right\}\right]-\theta \nabla \phi
$$

where $\Omega^{\alpha}$ is the thermal equivalent part of resolved shear stress $\tau^{\alpha}, w_{a}$ is the parameter which describes the strain (and depends on dislocation density), $\mu_{a}$ is the modulus of rigidity, $\beta_{a}$ is the lattice defect energy parameter, and $\omega^{\alpha}$ is a parameter which depends on slip resistance of $\alpha$-slip system. The three terms within the square bracket of Equation (20) represent the contribution of mechanical, thermal and lattice defect energies, respectively, to the energy dissipated during the deformation.

The crystal plasticity model developed above is numerically solved using finite element method. The algorithm followed is given in Table $\mathbf{1}$ and is implemented in ABAQUS using a user defined subroutine.

\section{Numerical Modeling}

Finite element simulations are done for polycrystalline austenitic, martensitic and two-phase high-Mn steel used for pipes in well applications. The two-phases considered are face centered cubic (FCC) austenite and body centered cubic (BCC) martensite. Both crystals are modeled as a single cubic finite element using 8-noded 3-D brick element with reduced integration (C3D8R), using one point integration rule. Each finite element represents 500 grains with random texture. In all cases, three primary deformation modes are considered i.e. uniaxial tension and compression, and simple shear. Figure 2 shows geometry of one element with prescribed loading and boundary conditions for tension, compression and shear. The element is a cube with each side of $1 \mathrm{~mm}$. For tension and compression modes, a displacement of $\pm 0.25 \mathrm{~mm}$ is applied on the face having normal in the $+e_{3}$ axis direction, and for simple shear displacement is applied in the $+e_{1}$ direction on the same surface (Figure 2). Planar symmetric boundary condition is applied on three faces while two remaining surfaces are traction free. The material models for austenitic and martensitic steels include material and hardening parameters. The material parameters are defined through their respective elasticity tensors, $\tilde{\mathbb{C}}^{a}$ and $\tilde{\mathbb{C}}^{m}$. The elasticity tensor elements for austenitic and martensitic steels are used in the current work as reported in [13]: $c_{11}^{a}=$ 286.6, $c_{12}^{a}=166.4, c_{44}^{a}=145.0$ and $c_{11}^{m}=372.4, c_{12}^{m}=$ $345.0, c_{44}^{m}=201.9$ (in GPa). Here, superscripts $a$ and $m$ represent austenite and martensite, respectively. The material parameters for dual-phase steels are represented by the effective elasticity tensor, $\tilde{\mathbb{C}}^{e}$ as given in Equation (13). The hardening parameters for austenitic and martensitic steels are extracted from the calibration of experimental results reported in [14], and given in Table 2.

\section{Results and Discussion}

Uniaxial tension, compression, and simple shear simulations have been performed in order to estimate the deformation behavior of steel with varying volume fraction of austenite and martensite phases. The simulations have been done for dual-phase steel having 5, 10, 15, 20, 25, and 50 percent of martensite. In uniaxial tension and compression, Cauchy stress component $T_{33}$ is plotted against logarithmic strain $L_{33}$ as other stress components are negligible. The results are shown in Figures

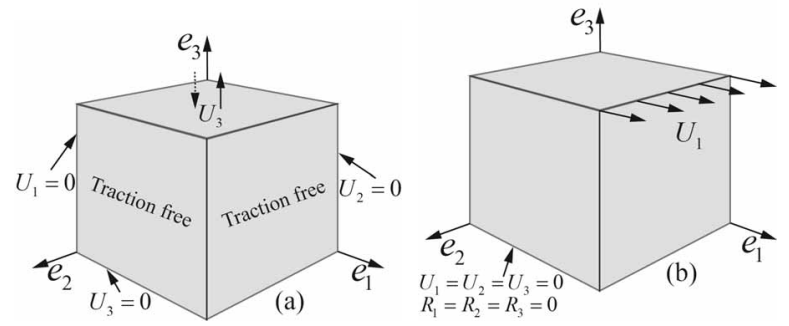

Figure 2. FE model geometry and boundary conditions: (a) Tension/compression, (b) Simple shear. 
Table 1. Numerical integration algorithm for crystal plasticity model.

1) START: Given parameters

2) Initial trial

Elastic deformation gradient

Green strain tensor

$2^{\text {nd }}$ Piola-Kirchhoff (PK) stress tensor

Slip resistance

Resolved shear stress

3) Iterative scheme to compute

3.1) Newton Raphson method to solve new estimate of

$$
\text { Compute shear strain rate }
$$

Compute plastic deformation gradient

Compute elastic deformation gradient

Compute Green strain tensor

Compute $2^{\text {nd }} \mathrm{PK}$ stress tensor

Check convergence:

3.2) Newton Raphson method to solve new estimate of

Compute slip resistance

Check convergence:

4) Update rotation tensor

5) Update Schmid tensor

6) Update Cauchy stress tensor

7) END: Output parameters

$$
F_{n}, F_{n+1}, F_{n}^{p}, S_{n}^{\alpha}, h_{0}, \dot{\gamma}_{0}, m, s_{\infty}, b
$$

$$
\begin{aligned}
& F_{n+1}^{e}=F_{n+1}\left(F_{n}^{p}\right)^{-1} \\
& E_{n+1}^{*}=\frac{1}{2}\left[\left(F_{n+1}^{e}\right)^{\mathrm{T}}\left(F_{n+1}^{e}\right)-I\right] \\
& T_{n+1}^{*}=\tilde{\mathbb{C}}: E_{n+1}^{*} \\
& s_{n}^{\alpha}
\end{aligned}
$$

$$
\tau_{n+1}^{\alpha}=\left(F_{n+1}^{e}\right)^{T}\left(F_{n+1}^{e}\right) T_{n+1}^{*} S_{n}^{\alpha}
$$

$T_{n+1}^{*}, s_{n+1}^{\alpha}$

$T_{n+1}^{*}$

$\dot{\gamma}_{n+1}^{\alpha}=\dot{\gamma}_{0}\left|\tau_{n+1}^{\alpha} / s_{n}^{\alpha}\right|^{1 / m} \sin \left(\tau_{n+1}^{\alpha}\right)$

$F_{n+1}^{p}=\left[I+\sum_{\alpha=1}^{N_{S L}} \dot{\gamma}_{n+1}^{\alpha} S_{n}^{\alpha}\right] F_{n}^{p}$

$F_{n+1}^{e}=F_{n+1}\left(F_{n+1}^{p}\right)^{-1}$

$E_{n+1}^{*}=\frac{1}{2}\left[\left(F_{n+1}^{e}\right)^{\mathrm{T}}\left(F_{n+1}^{e}\right)-I\right]$

$T_{n+1}^{*}=\tilde{\mathbb{C}}: E_{n+1}^{*}$

if $\left\|\Delta T_{n+1}^{*}\right\|_{2}<$ Tol , GOTO step 3.2 ELSE 3.1

$S_{n+1}^{\alpha}$

$\dot{S}_{n+1}^{\alpha}=\dot{S}_{n}^{\alpha}+\sum_{\beta=1}^{N_{S L}} q^{\alpha \beta} h_{0}\left(1-s_{n}^{\beta} / s_{\infty}\right)^{b}\left|\dot{\gamma}_{n+1}^{\beta}\right|$

if $\left\|\Delta s_{n+1}^{\alpha}\right\|_{2}<$ Tol , GOTO step 4 ELSE 3.1

$R_{n+1}^{e}=\exp \left(-\Delta t \sum_{\alpha=1}^{N_{S L}} \dot{\gamma}_{n+1}^{\alpha} \cdot \operatorname{skew}\left(S_{n}^{\alpha}\right)\right) R_{n}^{e}$

$S_{n+1}^{\alpha}=R_{n+1}^{e} S_{n}^{\alpha}\left(R_{n+1}^{e}\right)^{\mathrm{T}}=m_{n+1}^{\alpha} \otimes n_{n+1}^{\alpha}$

$T_{n+1}=\left(F_{n+1}^{e}\right)\left\{\operatorname{det}\left(F_{n+1}^{e}\right) T_{n+1}^{*}\right\}\left(F_{n+1}^{e}\right)^{\mathrm{T}}$

$S_{n+1}^{\alpha}, T_{n+1}^{*}, T_{n+1}$

Table 2. Hardening parameters for austenite and martensite.

\begin{tabular}{ccccccc}
\hline Phase & $m$ & $\dot{\gamma}_{0}\left(\mathrm{~s}^{-1}\right)$ & $h_{0}(\mathrm{MPa})$ & $s_{0}^{\alpha}(\mathrm{MPa})$ & $s_{\infty}(\mathrm{MPa})$ & $b$ \\
\hline Austenite & 0.02 & 0.001 & 280 & 165 & 340 & 2.5 \\
Martensite & 0.01 & 0.001 & 300 & 235 & 350 & 1.8 \\
\hline
\end{tabular}

3(a) and (b). In both loading conditions, the two main observations are that 1 ) stress-strain curves are higher, and 2) hardening pattern (shape of the stress-strain curve) varies with increasing volume fraction of martensite in the austenite matrix. Higher stresses for higher martensite content are obviously as expected. As for hardening behavior, 

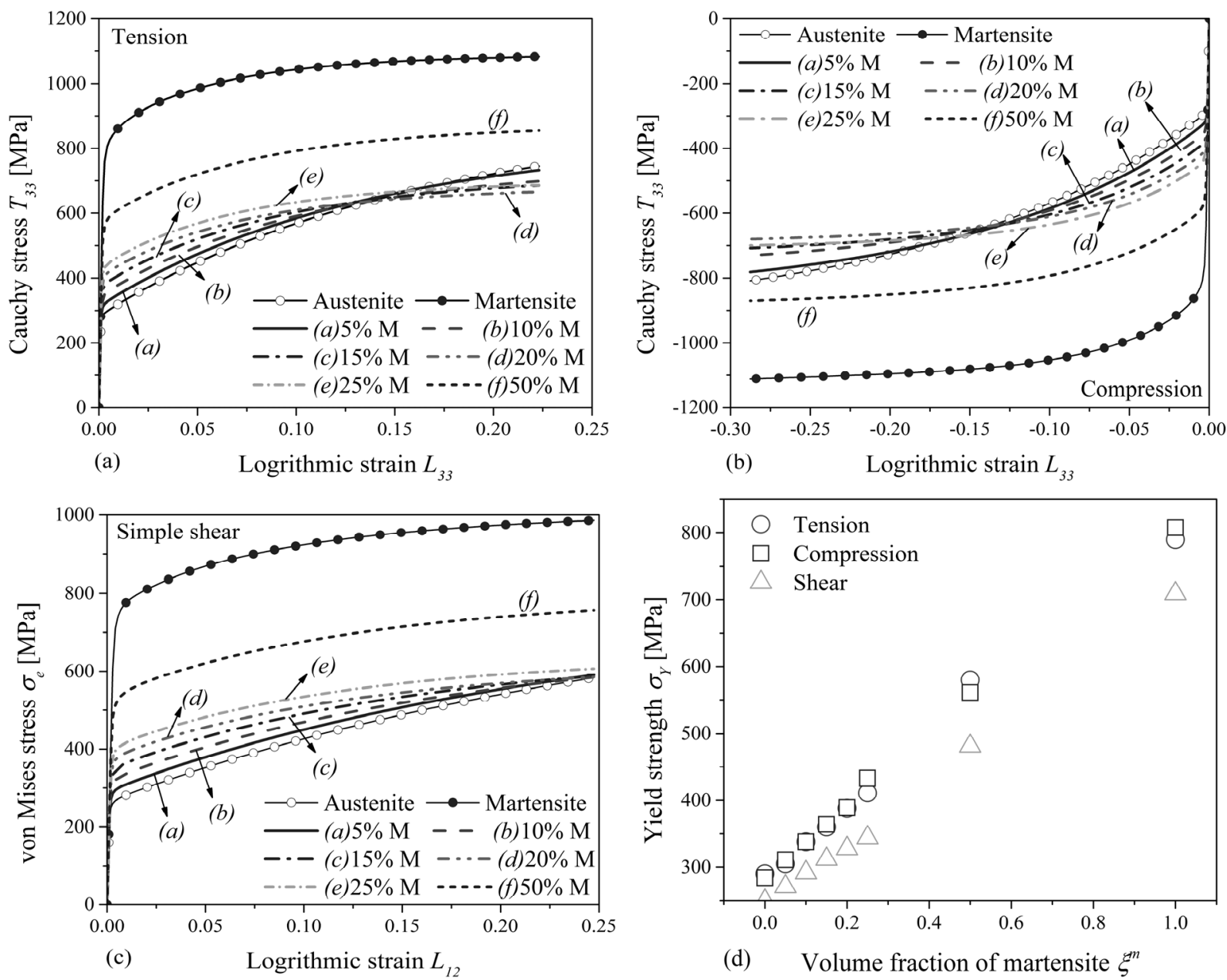

Figure 3. Stress strain behavior: (a) Tension; (b) Compression; (c) Shear; (d) Yield strength variation.

the hardening pattern (shape of the curve) of 5\%-martensite dual-phase alloy is almost the same as that of austenite; for higher martensite fraction (10\% or more) the curves are more like pure martensite. One very interesting observation is that all dual-phase curves coincide at a strain value of about 0.15 .

Figure 3(c) shows the variation of von Mises stress with logarithmic strain component $L_{12}$ for austenitic, martensitic and dual-phase steels in simple shear loading condition. At low magnitudes of strain, stress levels of steels having 5\% - 20\% of martensite are somewhat higher than pure austenite; but above the strain value of 0.2 these dual-phase steels show almost the same stress magnitude as that of austenitic steel. As observed for tension-compression, dual-phase alloys with $25 \%$ or more martensite show hardening behavior that is closer to martensite.

The variation in yield strength under all three loading conditions (tension, compression, shear) is almost linear; Figure 3(d). Yield strength values are almost identical under tension and compression at martensite fraction of up to $20 \%$. For higher amounts of martensite, there is a difference of about $20 \mathrm{MPa}$ in yield strengths under ten- sion and compression. An increase in yield strength of an alloy with increasing martensite content (harder phase) is as expected. However, the difference in yield strength behavior under tension and compression for higher martensite content may be due to various reasons: 1) increase in heterogeneity due to random distribution of harder phase, 2) mismatch in the crystal structure from FCC to BCC, which results in changing the slip deformation mechanism, 3) dislocation pile up at the grain and austenitemartensite boundaries, which may delay the onset of yielding, etc.

The magnitude of dissipated energy for austenitic, martensitic and dual-phase alloys for the three deformation modes is illustrated in Figure 4. As expected, martensitic steel (hardest and strongest phase) needs the highest amount of energy required to produce the same magnitude of permanent strain. The other steel alloys with different volume fraction of martensite show intermediate values of dissipated energy. It is interesting to note that under tension, Figure 4(a), the dissipated energy of dual-phase alloys reduces after reaching equivalent strain of 0.2 , except for $5 \%$ martensite. This trend is not observed in the 

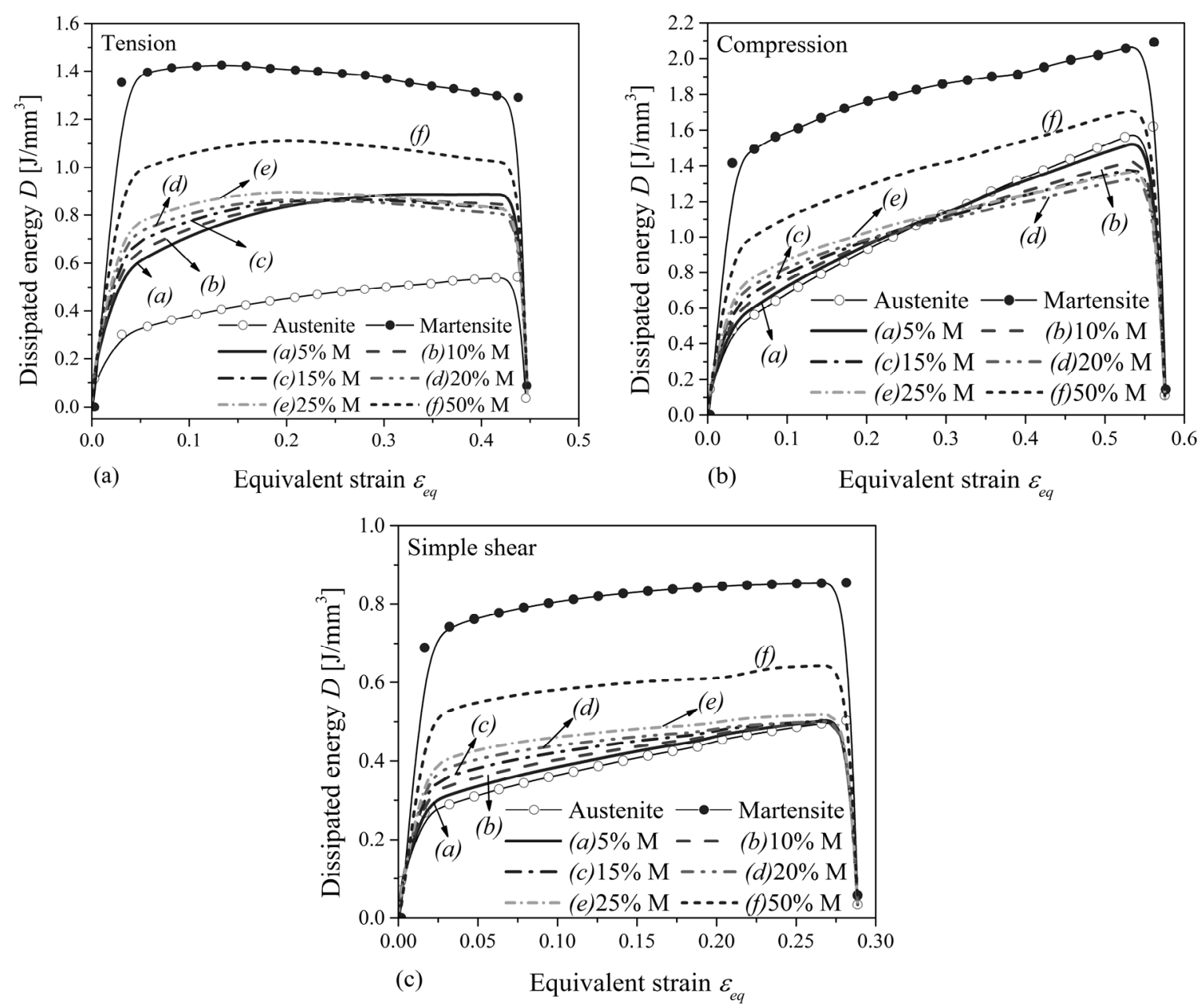

Figure 4. Dissipated energy variation of dual-phase steels: (a) Tension; (b) Compression; (c) Shear.

case of compression or shear. This behavior could be due to the interaction among slip systems (latent hardening), which is responsible for hardening and softening of a metallic material during the course of deformation. In compression and shear, there is a high possibility for slip systems to interact more than in tension because of the material flow behavior. This produces more hardening effects as a result of dislocation interlocking and dislocation pileup at the grain boundary or within the grain. The energy required to deform the material permanently therefore becomes higher as the deformation progresses under compression and shear. It can also be seen (Figure 4) that under tensile loading there is a significant difference in dissipated energies of austenitic, martensitic and rest of the dual-phase steels. This trend is less prominent in compression and shear. These observations show that the addition of harder martensite phase in an austenite matrix may give significant variation in material deformation behavior subjected to different loading conditions.

All of the above results can be crucial in the applications of dual-phase alloy steels where large permanent deformation is required. This investigation becomes even more important when the material needs to be deformed using less energy. Prime examples are the use of expandable tubulars in the oil and gas industry, extrusion of seamless steel pipes, rolling of aluminum thin sheets, etc.

\section{Conclusion}

A mathematical model has been developed for the elasticplastic deformation of austenitic, martensitic, and austenitic-martensitic steels, and a numerical investigation has been carried out for their behavior under tension, compression and shear. The percentage of martensite in dual-phase alloy is varied from $5 \%$ to $50 \%$. Under tension and compression, $5 \%$ martensite steel behaves almost like austenite, while two-phase steels having $10 \%$ or more martensite exhibit hardening behavior (shape of stress-strain curve) that is closer to martensitic steel. Loaded under shear, stress levels of steels having $5 \%$ - $20 \%$ of martensite are somewhat higher than pure austenite; but above the strain value of 0.2 , these two-phase steels show almost the same stress magnitude as that of austenitic steel. Variation of yield strength against martensite content is almost linear 
under all loading conditions. Being the hardest and strongest, martensitic steel needs the highest amount of energy required to produce a given amount of permanent strain, while two-phase alloys require intermediate values of dissipated energy. Under tension, less dissipated energy is required after reaching a strain value of around $0.2-0.3$.

\section{REFERENCES}

[1] A. Filippov, R. Mack, L. Cook, P. York, L. Ring and T. McCoy, "Expandable Tubular Solutions," SPE Annual Technical Conference and Exhibition, Houston, 1999.

[2] T. Pervez, S. Z. Qamar, O. S. Al-Abri and R. Khan, “Experimental and Numerical Simulation of In-Situ Tube Expansion for Deep Gas-Wells,” Journal of Materials and Manufacturing Processes, Vol. 27, No. 7, 2011, pp. 727732. http://dx.doi.org/10.1080/10426914.2011.648037

[3] R. Kuziak, R. Kawalla and S. Waengler, "Advanced High Strength Steels for Automotive Industry," Archives of Civil and Mechanical Engineering, Vol. 8, No. 2, 2008, pp. 103-117. http://dx.doi.org/10.1016/S1644-9665(12)60197-6

[4] E. Jimenez-Melero, N. H. van Dijk, L. Zhao, J. Sietsma, S. E. Offerman, J. P. Wright and S. van der Zwaag, "Martensitic Transformation of Individual Grains in Low-Alloyed TRIP Steels," Scripta Materialia, Vol. 56, No. 5, 2007, pp. 421-424. http://dx.doi.org/10.1016/j.scriptamat.2006.10.041

[5] C. G. Lee, S.-J. Kim, T.-H. Lee and S. Lee, "Effects of Volume Fraction and Stability of Retained Austenite on Formability in a $0.1 \mathrm{C}-1.5 \mathrm{Si}-1.5 \mathrm{Mn}-0.5 \mathrm{Cu}$ TRIP-Aided Cold-Rolled Steel Sheet," Materials Science and Engineering: A, Vol. 371, No. 1-2, 2004, pp. 16-23. http://dx.doi.org/10.1016/S0921-5093(03)00035-2

[6] T. Pervez, R. Khan, S. Z. Qamar, F. Al-Jahwari, F. J. Sanchez and B. A. Abri, "Post-Expansion Characterization of Expandable Tubular: Progress and Challenges,”
SPE/IADC Middle East Drilling Technology Conference and Exhibition, Muscat, 2011.

[7] V. I. Levitas and I. B. Ozsoy, "Micromechanical Modeling of Stress-Induced Phase Transformations. Part 1. Thermodynamics and Kinetics of Coupled Interface Propagation and Reorientation,” International Journal of Plasticity, Vol. 25, No. 2, 2009, pp. 239-280. http://dx.doi.org/10.1016/j.ijplas.2008.02.004

[8] ABAQUS, “Software Version 6.11,” Dassault Systèmes, 2012.

[9] E. H. Lee, "Elastic-Plastic Deformation at Finite Strains," Journal of Applied Mechanics, Vol. 36, No. 1, 1969, pp. 1-6. http://dx.doi.org/10.1115/1.3564580

[10] S. R. Kalidindi, C. A. Bronkhorst and L. Anand, "Crystallographic Texture Evolution in Bulk Deformation Processing of FCC Metals,” Journal of Mechanics and Physics of Solids, Vol. 40, No. 3, 1992, pp. 537-569. http://dx.doi.org/10.1016/0022-5096(92)80003-9

[11] R. J. Asaro and A. Needleman, "Texture Development and Strain Hardening in Rate Dependent Polycrystals," Acta Metallurgica, Vol. 33, No. 6, 1985, pp. 923-953. http://dx.doi.org/10.1016/0001-6160(85)90188-9

[12] R. Khan, "Micromechanical Modeling of Crystal and Transformation Plasticity in Multiphase Steels," Ph.D Thesis, Mechanical \& Industrial Engineering Department, Sultan Qaboos University, Muscat, 2013.

[13] S. Turteltaub and A. S. J. Suiker, "A Multiscale Thermomechanical Model for Cubic to Tetragonal Martensitic Phase Transformations,” International Journal of Solids and Structures, Vol. 43, No. 14-15, 2006, pp. 4509-4545. http://dx.doi.org/10.1016/j.ijsolstr.2005.06.065

[14] T. Narutani, G. B. Olson and M. Cohen, "Constitutive Flow Relations for Austenitic Steels during Strain-Induced Martensitic Transformation,” Journal De Physique IV, Vol. C4, No. 12, 1982, pp. 429-434. 